\title{
IMITATING NEUTRON IRRADIATION DAMAGE USING A COMBINATION OF ION BEAMS
}

\author{
VÁClAV Š́́sL ${ }^{a, *}$, MARTIN ŠEVEČEK ${ }^{a, b}$ \\ ${ }^{a}$ Department of Nuclear Reactors, Faculty of Nuclear Sciences and Physical Engineering, Czech Technical \\ University in Prague, V Holešovičkách 2, Prague 8, Czech Republic \\ ${ }^{b}$ Department of Nuclear Science and Engineering, Massachusetts Institute of Technology, 77 Massachusetts Ave, \\ Cambridge, MA, USA \\ * corresponding author: sislvacl@fjfi.cvut.cz
}

ABSTRACT. There is a strong motivation for using ion beams to imitate neutron irradiation damage, mainly in order to reduce costs and time demands linked to neutron irradiation experiments. The long-term goal of the authors is to create an ion irradiation methodology, which could be employed in the development process of innovative nuclear fuel materials. This methodology will be based on combining of a set of ion beams in such a way that the final distribution of irradiation-induced defects in the material structure is similar to the one which would have been introduced by neutrons in a nuclear reactor.

The first part of the methodology is represented by an optimization tool described here. The tool uses a third party Monte Carlo code SRIM to simulate ion transport in a target and to determine the distribution of radiation damage. Subsequently, a custom genetic optimization algorithm is applied to a set of damage distribution profiles to find their optimal combination.

KEYwORDS: Accident tolerant fuel, fuel cladding, radiation damage, SRIM, defects distribution optimization, genetic algorithms.

\section{INTRODUCTION}

Development of innovative materials for nuclear reactor applications (such as new cladding materials) is a complex multidisciplinary process. One of the criteria to be fulfilled is to prove resistance of the material against neutron irradiation and to quantify material characteristics that degrade with radiation. A traditional approach of proving irradiation tolerance is through irradiation in a research reactor and subsequent post-irradiation experimental testing (PIE). However, using a research reactor for irradiation purposes has multiple disadvantages, of which the most significant ones are time demands and costs.

The time demands are the result of two factors. First, the irradiation time itself is long, as the damage rate in a nuclear reactor is usually low, thus it takes a long time to accumulate the desired damage (often several months or even years). Second, since the samples become radioactive, further time is needed for decaying of the samples before subsequent testing. The fact that samples become radioactive also results in requirements for special handling procedures and facilities certified for such purposes. Another drawback of neutron irradiation is the need for designing of special capsules, in which the samples are placed during irradiation or need to handle the material in hot cells. This further increases the costs and complexity of the experiments.

There are also advanced nuclear reactors under development around the world. The high dose reactor

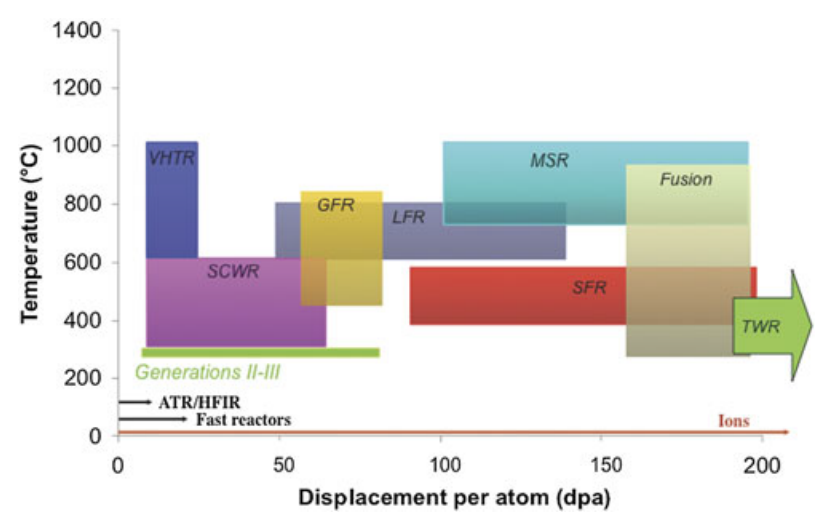

Figure 1. Comparison of approximated requirements for temperature and dpa for advanced reactor concepts and annual damage rates produced by current research and fast reactors. 1

concepts as molten salt reactors or traveling wave reactor are very challenging from the perspective of radiation damage of structural components that must survive radiation damage up to $250 \mathrm{dpa}$ or higher. 1] This makes the development of new materials very complicated and leads to excessive prolongation of the development of advanced reactors. The requirements for temperature and level of radiation damage to be reached for different advanced reactors are summarized in Figure 1

Taking into account all the problems linked to neutron irradiation in research reactors, there is a strong motivation for using ion beams. The main advantages 
of ion irradiation can be summarized as:

- Sources of ion beams (accelerators) are widelyspread.

- Dose rates are much higher compared to neutron irradiation, thus exposure times are as low as hours or tens of hours.

- There is no need for special capsule design.

- There is only a low or none residual activity of irradiated samples.

- Experiment conditions can be well controlled - easily adjustable ion energy, dose rate, and sample temperature.

- Damage cascades produced mainly by Primary Knock-on Atom (PKA) in the case of irradiation by heavy ions of typical energies are similar to those created by a PKA knocked by energetic neutrons.

There are, however, differences between the effects of neutron and heavy ion irradiation. These will be discussed in the following section. The goal of current research is to achieve as comparable irradiation damage profile as possible resulting in imitation of irradiation damage by heavy ions.

\section{DifFERENCES IN EFFECTS OF ION AND NEUTRON IRRADIATION}

In spite of the fact that ion irradiation offers significant advantages compared to neutron irradiation, there is a fundamental question to be answered: Are the effects of ion irradiation comparable to those created by neutrons?

According to Was [2], even though there are significant differences between the direct effects of neutron and ion irradiation, it is possible, to a certain degree, imitate the effects of neutron irradiation using ion beams. An important fact is that it is the final state of the material that determines the equivalence, not the path taken.

The main differences between effects of ion and neutron irradiation are [1]

- Particle energy spectrum:

Neutrons in a reactor have a wide energy spectrum ranging between several orders of magnitude from thermal energies $(0.025 \mathrm{eV})$ up to fast neutrons $(\mathrm{MeVs})$. On the other hand, heavy ions produced by an accelerator have very narrow range of energy (usually considered as monoenergetic beams).

- Penetration depth and damage distribution: Neutron's mean free path in structural materials of nuclear reactors is in order of centimeters, thus they can penetrate deep in a target. A large mean free path also means that the spatial damage distribution created by neutrons is homogeneous over many millimeters of the penetration depth. Ions, on the other hand, due to their electric charge, lose energy much faster when traveling through a material

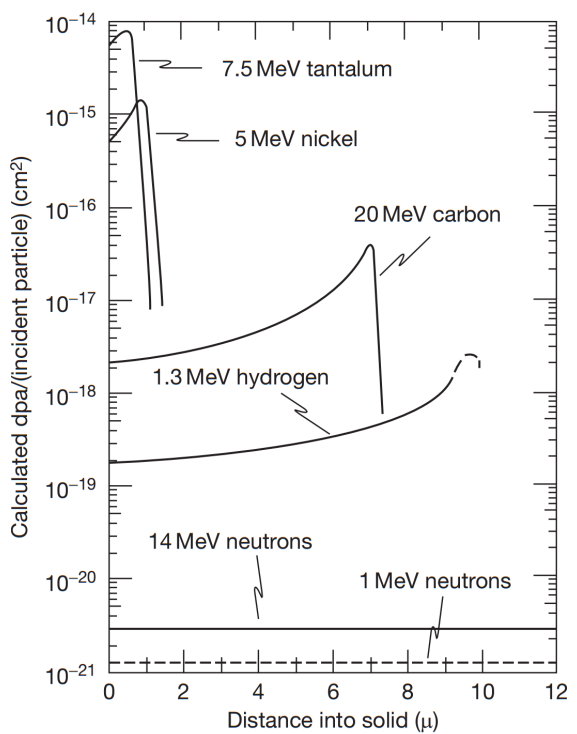

FIGURE 2. Damage distributions caused by various energetic particles. 2]

and their penetration depths are orders of magnitude shorter. They usually penetrate between 0.1 and $100 \mu \mathrm{m}$ depending on the particle type and its energy, see Figure 2.

- Damage rate:

As the cross-section for ion-atom interaction is much higher compared to neutron-nuclear interaction, the damage rate relative to the particle fluence is orders of magnitude higher for ion irradiation than for neutron irradiation.

- Nature of induced defects:

Neutrons and heavy ions produce large damage clusters of comparable size and nature. Light ions and protons produce smaller clusters or isolated point defects and electrons produce widely separated self-standing point defects. This behavior is more complex due to diffusion related effects that are closely linked to the temperature of the target.

In the presented study, we decided to tackle the issue of different damage distributions resulting from neutron and ion irradiation. Our goal was to combine a set of several ion beams of different energies and/or ion types. Assuming that the damage profiles created by individual beams can be summed-up independently, it is then possible to find a particular optimized combination leading to a relatively flat damage profile similar to the one, which would have been created by neutrons. The proposed optimization method is described in the following section, an example of a typical damage profile created by a single ion beam (6.0 MeV, Ni) and an optimized damage profile, as we defined it, are shown in Figure 3

In the past, there were multiple studies investigating a possibility to combine irradiation by multiple ion beams to mimic neutron irradiation [3 5]. However, the main aim was not to obtain a uniform damage 

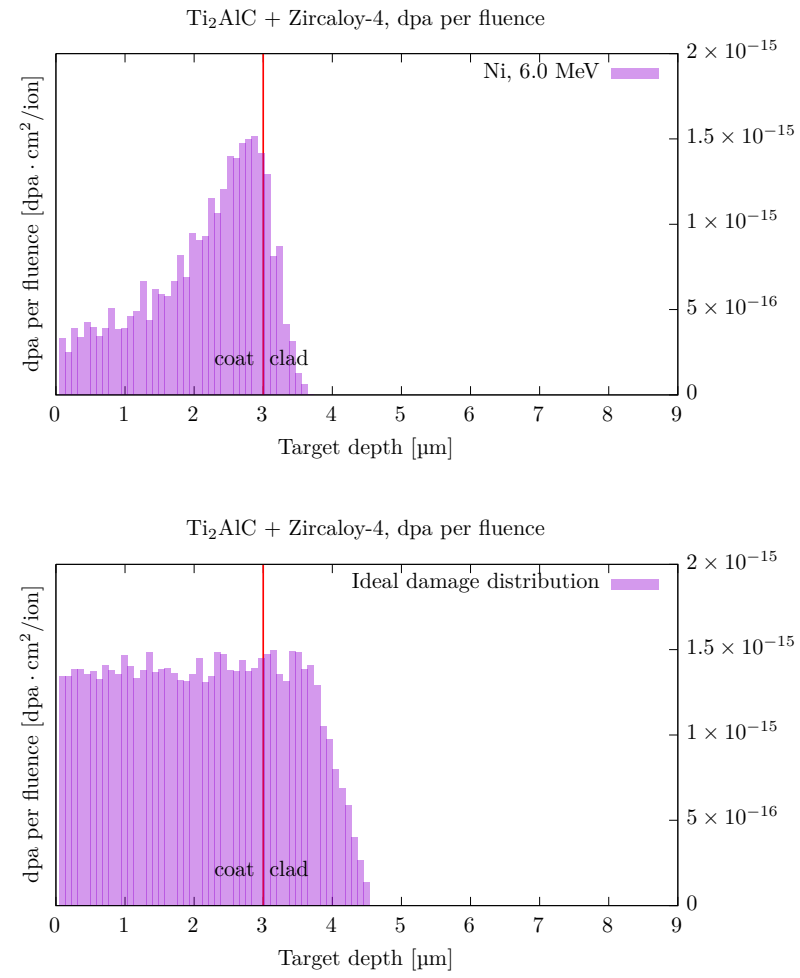

FiguRE 3. Damage profile created by $6 \mathrm{MeV}$ nickel ions irradiating $3 \mu \mathrm{m}$ thick $\mathrm{Ti}_{2} \mathrm{AlC}$ coating layer deposited on Zircaloy-4 slab (top) and an optimal damage profile as defined by the authors (bottom).

distribution, but rather to address neutron-induced transmutation by injecting He while irradiating by another type of ions to obtain the desired level of damage. Also, a procedure combining neutron irradiation for obtaining radiation damage and He irradiation for injecting bubbles is being used by the community. 6]

The possibility of obtaining a layer ${ }^{1}$ of evenly irradiated material might be especially suitable for testing Accident Tolerant Fuel (ATF) multi-component cladding materials. During such tests, it is needed to evaluate properties under irradiation of a thin coating layer deposited on a material, which irradiation properties are already well known (Zr-based alloys serving as substrates). Another objective is to test the behavior of the interface substrate/coating. Structural changes at the interface caused by radiation might lead to issues related to adhesion of the coatings, cracking or excessive stress in the ATF cladding.

Neutron irradiation will be always required to qualify materials for new reactor applications, however ion irradiation, as a low-cost and fast damage method, can serve as a screening method for evaluating new material concepts. With the use of ion irradiation, the development process of new materials for current and future reactor applications can be much faster and cheaper.

\footnotetext{
${ }^{1}$ Relatively thin, limited by a penetration depth of the beams.
}

\section{Methodology And example RESULTS}

The proposed optimization method will be demonstrated on an example of irradiation of a thin $\mathrm{Ti}_{2} \mathrm{AlC}$ (MAX phase) coating deposited on Zircaloy-4 alloy. Zircaloy-4 is a standard binary Zr-Sn alloy used by the nuclear industry for decades. The MAX phase coating is proposed as a potential candidate material enhancing the safety features of the standard Zr-based cladding. The ultimate goal is to irradiate the coating layer with multiple ion beams in such proportion that the sum of their respective damage profiles will result in an optimal damage distribution.

The optimal damage distribution is defined as a flat distribution. That means a situation, where at each point of the depth of the target, there is the same irradiation-induced damage expressed by the quantity dpa per fluence $\left[\mathrm{dpa} \cdot \mathrm{cm}^{2} / \mathrm{ion}\right]$. Only the damage distribution in the coating layer of the target is taken into account, the shape of the damage profile in the substrate (Zircaloy-4) is not considered. However, it should be noted that for the purposes of irradiation experiments, it is crucial for the beam to be able to penetrate the full depth of the coating, since the behavior of the interface between the coating and the bulk cladding is of a significant concern in the development of multi-component cladding materials. For that reason, the thickness of the coating needs to be optimized to fit the requirements of the test.

In general, an arbitrary number of beams can be used for the optimization. There are two basic conditions the beams shall fulfill: First, at least one of the beams should be able to penetrate the full width of the coating layer. Second, the Bragg peaks of damage distributions created by individual beams should not overlap significantly. For demonstration purposes, it is assumed here that four different ion beams are to be combined, namely: $\mathrm{Fe}(6 \mathrm{MeV})$ and $\mathrm{Ni}(6,3.4$, and $1.7 \mathrm{MeV})$. Each of these beams creates a unique damage profile ${ }^{2}$ when irradiating the target. These profiles are denoted as $A(z), B(z), C(z)$, and $D(z)$, respectively, and are functions of $z$ - the penetration depth inside the target. These profiles can be also represented as vectors $A=\left\{A_{1}, A_{2}, \ldots A_{N_{B}}\right\}$, $B=\left\{B_{1}, B_{2}, \ldots B_{N_{B}}\right\}, C=\left\{C_{1}, C_{2}, \ldots C_{N_{B}}\right\}$, and $D=\left\{D_{1}, D_{2}, \ldots D_{N_{B}}\right\}$, where $N_{B}$ is the number of bins present in the coating part of the target.

The aim is to find such parameters $a, b, c, d$ fulfilling the fundamental condition

$$
a+b+c+d=1
$$

so that the resulting damage profile

$$
f_{j}=a A_{j}+b B_{j}+c C_{j}+d D_{j}, j \in\left\{1, \ldots N_{B}\right\}
$$

will be as flat as possible.

\footnotetext{
${ }^{2}$ Damage profiles can be obtained using Monte Carlo simulation tools, e.g. SRIM [7] as in the case of this study.
} 
The flatness of the distribution can be described by its standard deviation. A small standard deviation corresponds to an even distribution of the defects, optimal (flat) distribution having the standard deviation equal to zero. The standard deviation of a damage distribution $f$ is calculated as

$$
S D E V=\sqrt{\frac{1}{N_{B}-1}\left[\frac{S_{2}}{N_{B}}-\left(\frac{S_{1}}{N_{B}}\right)^{2}\right]},
$$

where $N_{B}$ is the number of bins present in the coating part of the target and

$$
\begin{gathered}
S_{1}=\sum_{j=1}^{N_{B}} f_{j}=\sum_{j=1}^{N_{B}} a A_{j}+b B_{j}+c C_{j}+d D_{j}, \\
S_{2}=\sum_{j=1}^{N_{B}} f_{j}^{2}=\sum_{j=1}^{N_{B}}\left(a A_{j}+b B_{j}+c C_{j}+d D_{j}\right)^{2} .
\end{gathered}
$$

Having defined the problem and the measure of quality of a solution of this problem, standard optimization tools can be employed. In this case, a proprietary implemented genetic optimization algorithm [8] was used to find an optimal set of parameters $a, b, c, d$.

Genetic algorithms work with five basic concepts: generation, population, individual, genotype, and gene.

- Each generation is represented by one population.

- A population contains individuals, who represent possible solutions of the problem.

- Each individual is represented by its genotype - a set of genes.

- A gene can be a binary variable, an integer, a float or anything else.

The algorithm iterates over generations with an aim to transfer the best individuals from the current generation to the following one. The quality of an individual is expressed by the value of its fitness function, the higher the fitness, the better the individual. A flowchart describing the genetic algorithm used for irradiation optimization is showed in Figure 5

At the beginning, damage profiles are loaded from previously prepared files. (See number 1 in the Figure 5.) An initial population is created using the method initialize_population as a set of random individuals. (Nr. 2 in Figure 5) For each individual, the genotype (values $a, b, c, d$ ) is set as an array of random numbers from an interval $\langle 0,1\rangle$. Eventually, the genotype is normalized so that the individual fulfills the condition (1).

After creating the initial population, an iteration over the number of generations begins (nr. 3). Following steps are executed in each iteration.
- The quality of each individual in the population is calculated (nr. 4). Individual's quality is represented by the fitness function value. Since the algorithm is searching for an individual with the highest fitness value, while our aim is to minimize the value $S D E V$, the fitness of an individual is calculated as

$$
F I T N E S S=\frac{1}{S D E V} .
$$

- A linear transformation is then applied on the fitness of each individual by subtracting the fitness of the worst individual in the population (nr. 5). The transformation improves the process of roulette selection, which will follow later. It ensures that high-quality individuals will be more likely to be selected for the process of crossing.

- The index of the best individual in the population is saved. The best individual will be eventually artificially inserted into the new population (elitism).

- A new population is created as an empty array (nr. $6)$. An iteration over a half of the number of individuals in each population (population_size/2) begins. Following steps are executed in each iteration.

- Two individuals (parents) are randomly selected (nr. 7).

- With the probability (1 - crossing_rate) the parents are directly inserted into the new population (nr. 8), with the probability crossing_rate, they undergo the process of crossing (nr. 9).

- In the crossing process, two new individuals (descendants) are created via random combination of genotypes of the parents.

- After the crossing process, each gene of each descendant is, with a probability (1 mutation_rate) left as it is (nr. 10), or with a probability mutation_rate modified (nr. 11) by addition of a random value from the distribution

$$
g(x, y)=0.01 \sqrt{-2 \ln (x)} \sin (2 \pi y),
$$

where $x$ and $y$ are random values from an interval $\langle 0,1\rangle$. After adding a random value, it may happen that the value of a gene is negative. In that case, the negative value is replaced by zero.

- The genotype of each descendant is normalized (nr. 12) so that he sum of all genes is equal to 1 .

- After the process of crossing (and mutation, in some cases), both descendants are inserted into the new population (nr. 13).

- After population_size/2 iterations, the new population is completed (nr. 14). 

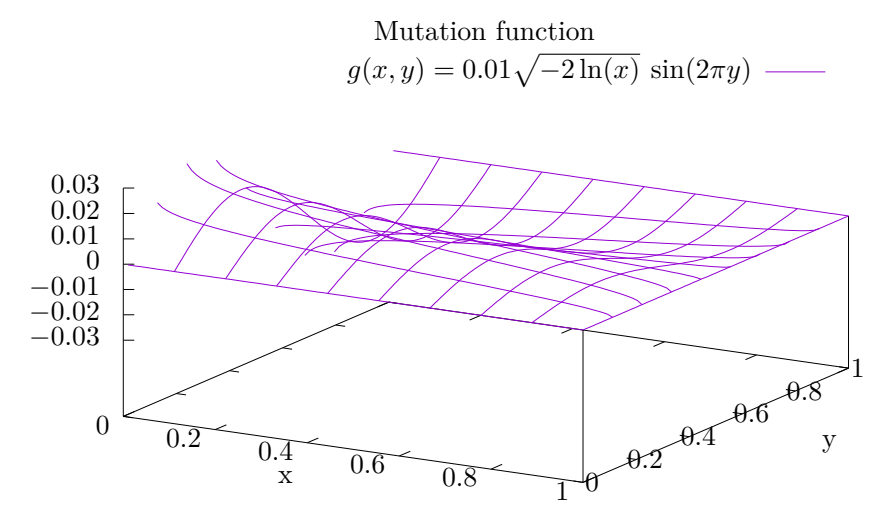

Figure 4. Two-dimensional mutation function used in the algorithm.

- In the last step of the loop, the best individual from the previous population is artificially inserted into the new population and replaces the first individual created through natural selection. This method is called elitism (nr. 15) and is executed regardless of whether or not the best individual from the previous population is actually better than the one it replaces.

The process of creating new populations based on crossing individuals from the previous one is repeated until a generation counter reaches a set value (nr. 16). After the loop terminates, the best individual is printed to the console (nr. 17) and the program is terminated.

The described algorithm was applied on the previously mentioned case: irradiation of a thin layer of $\mathrm{Ti}_{2} \mathrm{AlC}$ coating deposited on Zircaloy-4. This material is widely studied as a prospective candidate for the so-called Accident Tolerant Fuel (ATF) [9]. A visualization of the optimized damage profile obtained as a combination of four ion beams, Fe $(6 \mathrm{MeV})$ and $\mathrm{Ni}(6,3.4$, and $1.7 \mathrm{MeV})$, is shown in Figure 6. The ion beams (particles and their energy) were chosen based on available and widely used ion beams (e.g. [6]). The methodology and the algorithm are, however, very generally applicable. In fact, any combination of ion beams with any predefined energy can be used. The presented case serves only as an illustration of the presented method. The resulting parameters are: $a=0.05, b=0.48, c=0.22$, and $d=0.25$. The standard deviation was reduced by $98 \%$ compared to irradiation by a single beam $(\mathrm{Fe}, 6 \mathrm{MeV})$. The final values of parameters $a, b, c, d$ can be then used to design an irradiation test of the particular fuel concept.

The algorithm is very flexible and it allows users to optimize their own irradiation experiment. It can be easily modified based on available ions and their energies for a particular irradiation facility and particular material. There are certain simplifications compared to the real case. The recombination of defects and other temperature effects are neglected. Furthermore, the beams act as independent meaning that the algorithm treats them separately without any interaction between them and neglects combined effects. It is, however, difficult to estimate the influence of these phenomena on the presented results. As for the issue of defect diffusion during irradiation, it would be convenient to perform the irradiation at decreased temperatures as it would make the process of diffusion slower. The diffusion related phenomena of radiation effects were studied by 10 who proposed a correlation that can be used to account for the different damage rates when using different types of beams. When using ions or protons imitating irradiation damage inside LWR reactors, the temperature of the target should be increased by $15-40{ }^{\circ} \mathrm{C}$ compared to the temperature in reactor. Another approach might be to irradiate the sample with multiple beams at the same time not separately. In this case the defects are created uniformly, and the diffusion is slow due to a low defects concentration gradient in the material. This brings challenges to the operation of the accelerator and the experiment would be more complicated to perform.

\section{Discussion and Conclusions}

It is believed that ion irradiation can serve as an inexpensive and fast verification method for application of new materials in the nuclear industry. The methodology of ion irradiation can be optimized to cope with the fundamental differences between the primary effects of ion and neutron irradiation as was shown in this paper. However, ion beam testing is unlikely to replace neutron irradiation in research reactors for the purposes of nuclear fuel licensing.

The proposed method proved to deliver desired results - a homogeneous distribution of irradiation damage in a layer, which is relatively thin, but thick enough to be used for testing ATF multi-component cladding materials. On the other hand, a weakness of proposed solution is that only the issue of damage distribution was considered. All the other aspects, 


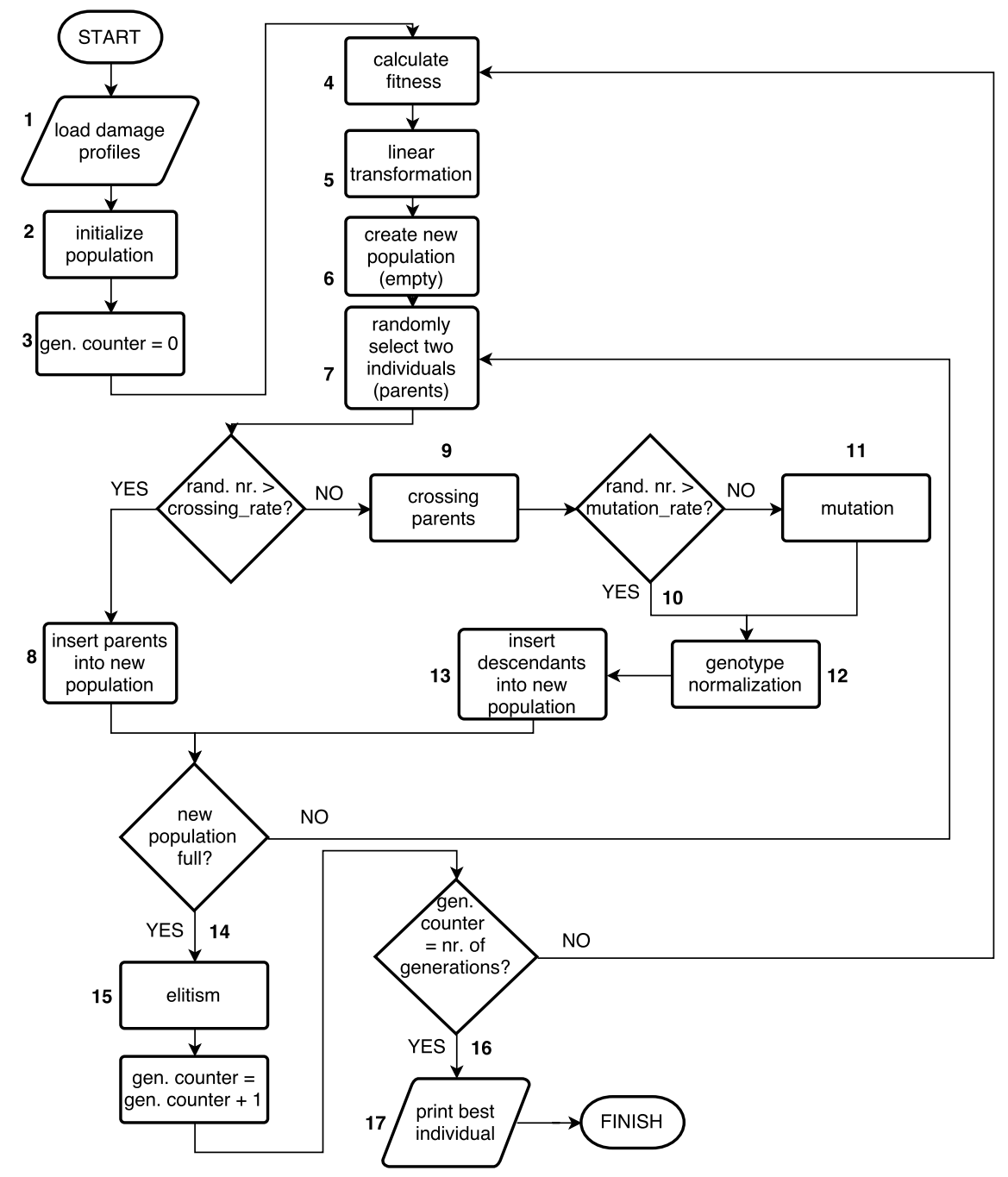

FigURE 5. Flowchart describing the genetic algorithm used to optimize radiation damage distribution using multiple ion beams.

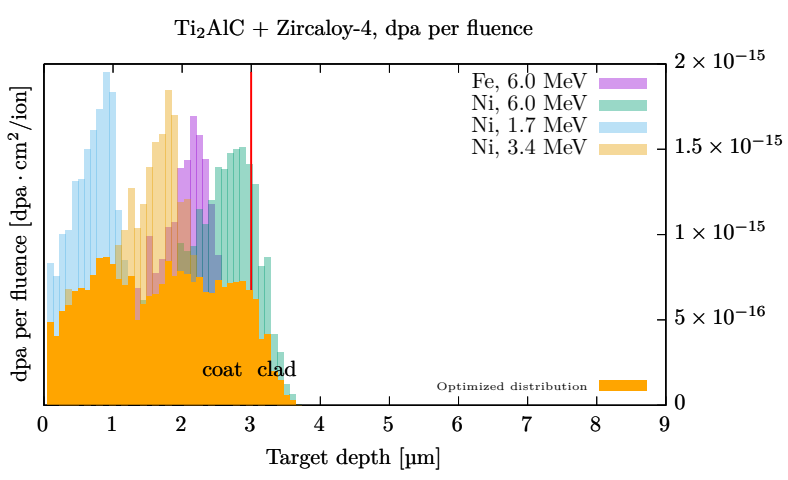

Figure 6. Damage profiles of four different ion beams irradiating $3 \mu \mathrm{m}$ thick $\mathrm{Ti}_{2} \mathrm{AlC}$ coating layer deposited on Zircaloy-4 slab (violet, green, blue and yellow) and their optimized combination (orange).

such as damage morphology, temperature differences or defects diffusion have not been taken into account.

It is, however, a perspective method enabling to imitate neutron irradiation using heavy ions. Planned irradiation experiments will be optimized using the proposed method and the resulting damage distribution will be more representative for the real application inside nuclear reactor cores compared to current simple heavy ion irradiation. The optimization tool and its manual can be found on the GitHub repository 8 and the application of this tool to real irradiation experiments with $\mathrm{Cr}$ and $\mathrm{CrN}$ coated cladding materials will be presented soon.

The $\mathrm{Cr}$ and $\mathrm{CrN}$ coated Zr-based alloys were irradiated also in the LVR-15 reactor in Rez up to 1 dpa. One of the goals of the proposed research is to study the advanced cladding material irradiated at the research reactor and compare the results directly to the irradiation of the same cladding concept from an accelerator with the same irradiation damage.

\section{LIST OF SYMBOLS}

ATF Accident Tolerant Fuel

DPA Displacements per Atom

PKA Primary Knock-on Atom

$S D E V$ Standard deviation 


\section{ACKNOWLEDGEMENTS}

The support from Student grant contest from Czech Technical University with code SGS16/252/OHK4/3T/14 is gratefully acknowledged. Many thanks belongs to my supervisor Ing. Martin Ševeček.

\section{REFERENCES}

[1] G. S. Was. Challenges to the use of ion irradiation for emulating reactor irradiation. Journal of Materials Research 30(09):1158-1182, 2015. DOI:10.1557/jmr.2015.73

[2] G. Was, R. Averback. Radiation damage using ion beams. Comprehensive Nuclear Materials 1:195, 2012.

[3] G. Was, Z. Jiao, E. Getto, et al. Emulation of reactor irradiation damage using ion beams. Scripta Materialia

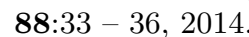

DOI:http://dx.doi.org/10.1016/j.scriptamat.2014.06.003

[4] A. Kohyama, Y. Katoh, K. Jimbo. Radiation damage study by advanced dual-ion irradiation methods. Materials Transactions 45(1):51-58, 2004.

[5] Y. Serruys, M.-O. Ruault, P. Trocellier, et al. Multiple ion beam irradiation and implantation: JANNUS project. Nuclear Instruments and Methods in Physics Research Section B: Beam Interactions with Materials and Atoms 240(1):124 - 127, 2005. Accelerators in Applied Research and Technology, DOI:http://dx.doi.org/10.1016/j.nimb.2005.06.100
[6] S. Taller, D. Woodley, E. Getto, et al. Multiple ion beam irradiation for the study of radiation damage in materials. Nuclear Instruments and Methods in Physics Research Section B: Beam Interactions with Materials and Atoms 412:1-10, 2017.

[7] R. Stoller, M. Toloczko, G. Was, et al. On the use of SRIM for computing radiation damage exposure. Nuclear Instruments and Methods in Physics Research Section B: Beam Interactions with Materials and Atoms 310:75 - 80, 2013. DOI:https://doi.org/10.1016/j.nimb.2013.05.008

[8] V. Š́sl. Optimization tool for ATF ion irradiation, GitHub repository. https://github.com/vsisl/vyzkumny_ukol, 2017.

[9] P. Xu, E. Lahoda, S. Ray, et al. Corrosion resistant coatings for zirconium-alloy cladding with improved accident tolerance. Nuclear Engineering and Technology 48:16-25, 2016.

[10] L. K. Mansur. Correlation of neutron and heavy-ion damage: II. The predicted temperature shift if swelling with changes in radiation dose rate $\mathbf{7 8}(1): 156-160$. DOI:10.1016/0022-3115(78)90514-7 